\title{
Positional expression profiling indicates candidate genes in deletion hotspots of hepatocellular carcinoma
}

\author{
Kathy Y-Y Chan ${ }^{1}$, Paul B-S Lai ${ }^{2}$, Jeremy A Squire ${ }^{3}$, Ben Beheshti ${ }^{3}$, Navy L-Y Wong ${ }^{1}$, \\ Shirley M-H Sy ${ }^{1}$ and Nathalie Wong ${ }^{1}$ \\ ${ }^{1}$ Department of Anatomical and Cellular Pathology, The Chinese University of Hong Kong, Shatin, NT, SAR \\ Hong Kong, China: ${ }^{2}$ Department of Surgery, The Chinese University of Hong Kong, Shatin, NT, SAR Hong \\ Kong, China and ${ }^{3}$ Department of Medical Biophysics and Laboratory Medicine and Pathobiology, University \\ of Toronto, Toronto, Ontario, Canada
}

\begin{abstract}
Molecular characterizations of hepatocellular carcinoma have indicated frequent allelic losses on chromosomes $4 q, 8 p, 16 q$ and $17 p$, where the minimal deleted regions have been further defined on $4 q 12-q 23,4 q 31-$ q35, 8p21-p22, 16q12.1-q23.1 and 17p13. Despite these regions are now well-recognized in early liver carcinogenesis, few underlying candidate genes have been identified. In an effort to define affected genes within common deleted loci of hepatocellular carcinoma, we conducted transcriptional mapping by highresolution cDNA microarray analysis. In 20 hepatocellular carcinoma cell lines and 20 primary tumors studied, consistent downregulations of novel transcripts were highlighted throughout the entire genome and within sites of frequent losses. The array-derived candidates including fibrinogen gamma peptide (FGG, at 4q31.3), vitamin D binding protein (at 4q13.3), fibrinogen-like 1 (FGL1, at 8p22), metallothionein 1G (MT1G, at 16q12.2) and alpha-2-plasmin inhibitor (SERPINF2, at 17p13) were confirmed by quantitative reverse transcriptionpolymerase chain reaction, which also indicated a more profound downregulation of FGL1, MT1G and SERPINF2 relative to reported tumor-suppressor genes, such as DLC1 (8p22), E-cadherin (16q22.1) and TP53 (17p13.1). In primary hepatocellular carcinoma examined, a significant repression of MT1G by more than 100fold was indicated in $63 \%$ of tumors compared to the adjacent nonmalignant liver $(P=0.0001)$. Significant downregulations of FGG, FGL1 and SERPINF2 were also suggested in 30,23 and $33 \%$ of cases, respectively, compared to their nonmalignant counterparts $(P<0.016)$. In summary, transcriptional mapping by microarray indicated a number of previously undescribed downregulated genes in hepatocellular carcinoma, and highlighted potential candidates within common deleted regions.
\end{abstract}

Modern Pathology (2006) 19, 1546-1554. doi:10.1038/modpathol.3800674; published online 15 September 2006

Keywords: hepatocellular carcinoma; deletion hotspots; cDNA array; transcriptional mapping

Hepatocellular carcinoma currently ranks the fifth most common malignancy worldwide. ${ }^{1,2}$ It carries a high cancer morbidity and mortality because by the time of clinical presentation, more than $80 \%$ of patients are at the advance inoperable stage..$^{2,3}$ The low efficacy of current screening regime by serum alpha-fetoprotein measurements and transabdominal ultrasound imaging has rendered most patients not being diagnosed in time for curative surgery.

Correspondence: Dr N Wong, DPhil, Department of Anatomical and Cellular Pathology, The Chinese University of Hong Kong, Prince of Wales Hospital, Shatin, NT, SAR Hong Kong, China.

E-mail: natwong@cuhk.edu.hk

Received 21 March 2006; revised 10 July 2006; accepted 18 July 2006; published online 15 September 2006
Thus, underpinning the genetic events associated with hepatocellular carcinoma is expected to improve our understanding on liver carcinogenesis and also potentially provide biomarkers that are of diagnosis and prognostic values.

Molecular studies using loss of heterozygosity analysis and comparative genomic hybridization have revealed recurrent chromosomal changes in hepatocellular carcinoma including losses on $1 \mathrm{p}$, 4q, 6q, 8p, 11q, 13q, 16q and 17p. ${ }^{4-6}$ As diminutions on $4 q, 8 p, 16 q$ and $17 p$ have been described in the nontumorous cirrhotic nodules and early preneoplastic liver dysplasia, these changes have been further implicated in the early carcinogenetic events of hepatocellular carcinoma. ${ }^{4-6}$ It has been long recognized that delineation of the smallest over- 
lapping regions in nonrandom genomic regions holds value in defining critical loci that are likely to harbor causative tumor-suppressor or protooncogene(s). In the case of hepatocellular carcinoma, only few tumor-suppressor genes have been described to the smallest overlapping regions. For example, deleted in liver cancer 1 (DLC1) on 8p21p22, ${ }^{7}$ E-cadherin (CDH1) on 16q12.1-q23.1 ${ }^{8}$ and tumor protein p53 (TP53) on $17 \mathrm{p} 13 .{ }^{9}$ Candidate genes in the smallest overlapping regions $4 q 12$ $\mathrm{q} 23^{10}$ and $4 \mathrm{q} 31-\mathrm{q} 35$, on the other hands, remains undescribed. ${ }^{11,12}$ As regions of aberrant genomic loci can harbor more than one tumor-related genes, such as RASSF1A, MLH1, TGFBR2 and BLU on chromosome $3 \mathrm{p} 21,,^{13-16}$ the existence of more than one tumor-suppressor gene within common deleted sites of hepatocellular carcinoma cannot be ruled out.

The microarray technology has greatly facilitated the investigation of whole genome expression analysis. Large-scale transcriptional profiling has so far been reported in multiple cancers including hepatocellular carcinoma. ${ }^{17-20}$ With regard to hepatocellular carcinoma, most microarray studies have emphasized on determining the profiles that allow differentiation of stepwise progressions from the preneoplastic lesions and in defining expression patterns associated with clinical subsets such as the prediction of drug resistance. ${ }^{21}$ Information on gene expression changes within regions of aberrant genomic loci, on the other hand, has been minimal. In this study, we have attempted to delineate the deregulated transcripts in regional imbalances of hepatocellular carcinoma, and especially in common deletions that have been described in the early carcinogenetic events. A detail investigation was undertaken using a high-resolution 19K cDNA microarray that allowed an average mapping resolution of $\sim 162 \mathrm{~kb}$ throughout the whole genome. Our array study examined a large panel of hepatocellular carcinoma cell lines that included early passages of hepatocellular carcinoma cultures and established cell lines. Analysis of cell lines can potentially increase detection sensitivity by minimizing secondary effects of the tumor microenvironments. However, despite cell lines represent a homogenous population of malignant hepatocytes; induced gene expressions from prolonged in vitro culture might correspond to a major inadequacy in identifying representative genes. To compensate for this potential limitation, we have also examined a cohort of primary hepatocellular carcinoma tumors, which is expected to provide representative results of clinical situation. Consistent downregulated transcripts found in both cell lines and tumors that were assigned to regions of deletion hotspots were further subjected to quantitative reverse transcription-polymerase chain reaction (qRT-PCR) validations. Results obtained were also compared to recognize tumor-suppressor genes such as DLC1, CDH1 and TP53.

\section{Materials and methods}

\section{Cell Lines}

Seven hepatocellular carcinoma cell lines, Hep3B, PLC/PRF/5, SNU387, SNU398, SNU423, SNU449 and SNU475, were obtained from the American Type Culture Collection (Rockville, MD, USA). According to recommendations, Hep3B and PLC/ $\mathrm{PRF} / 5$ were cultured in DMEM medium containing $10 \%$ fetal bovine serum (FBS) and $100 \mathrm{IU} / \mathrm{ml}$ penicillin and $100 \mathrm{U} / \mathrm{ml}$ streptomycin (Gibco BRL, Grand Island, NY, USA), while SNU387, SNU398, SNU423, SNU449, SNU475 were maintained in RPMI median containing $10 \% \mathrm{FBS}$ and $100 \mathrm{IU} / \mathrm{ml}$ penicillin and $100 \mathrm{U} / \mathrm{ml}$ streptomycin (Gibco BRL). Thirteen hepatocellular carcinoma cell lines (HKCI1 to 10 , and -C1 to C3) were established from our laboratory, eight of which HKCI-1, $-2,-3,-4,-5$ and HKCI-C1, -C2 and -C3 have been previously reported. ${ }^{22-25}$ Using the same methodology described, ${ }^{22-24}$ we have newly established five additional cell lines (HKCI-6, -7, -8, -9 and -10). The early passages 20-25 of the HKCI series of cell lines were utilized in the microarray study. These cell lines were maintained in complete medium containing RPMI 1640 glutamax with HEPES buffer supplemented with $10 \%$ FBS, $100 \mathrm{IU} / \mathrm{ml}$ penicillin, $100 \mathrm{U} / \mathrm{ml}$ streptomycin, $10 \mathrm{ng} / \mathrm{ml}$ selenium, $10 \mu \mathrm{g} / \mathrm{ml}$ transferrin and $10 \mu \mathrm{g} /$ $\mathrm{ml}$ insulin. All cultures were maintained in a humidified incubator at $37^{\circ} \mathrm{C}$ in an atmosphere of $5 \% \mathrm{CO}_{2}$.

\section{Patients}

Tumorous liver tissue was collected from 38 patients (aged 24-78 years, 84\% male) who underwent curative surgery for hepatocellular carcinoma at the Prince of Wales Hospital, Hong Kong. A corresponding adjacent nonmalignant liver tissue was secured for 30 patients. Informed consent was obtained from each patient recruited. Patients were predominantly hepatitis B carriers (95\%) with underlying liver cirrhosis indicated in $84 \%$ of cases. According to the American Joint Committee on Cancer tumor staging criteria, ${ }^{26}$ one case was graded as stage I, 20 cases as stage II, 11 as stage III and six as stage IV.

Microarray analysis was conducted on 20 hepatocellular carcinoma tumors, of which retrievable tumor RNA from 12 cases was further utilized in qRT-PCR validations. An additional 18 cases was further investigated by qRT-PCR, totaling 30 paired cases being assessed for the array-derived candidate genes.

\section{Microarray Analysis}

The expression array experiments were carried out according to the method previously described from 
our laboratory. ${ }^{25}$ Briefly, $10 \mu \mathrm{g}$ of Trizol extracted total RNA from cell lines and primary tumors was reverse-transcribed by AncT mRNA primer using Superscript II reverse transcriptase (Invitrogen, Carlsbad, CA, USA). A pool of three normal liver samples was used as reference purchased from three different companies (Ambion, Austin, TX, USA; Strategene, La Jolla, CA, USA; CloneTech, Pale Alto, CA, USA). Following fluorescence labeling of the transcribed cDNAs with Cy5-dCTP or Cy3-dCTP, the labeled cDNAs were mixed with calf thymus DNA, poly(dA), and yeast tRNA in Dighyb buffer (Roche Diagnostics, Mannheim, Germany) and hybridized onto cDNA microarray slides (Ontario Cancer Institute, Toronto, Canada). The $19 \mathrm{~K}$ cDNA microarray employed contains 19008 sequence-verified human genes and EST sequences that allowed an average resolution of $\sim 162 \mathrm{~kb}$ throughout the genome. Hybridization was carried out at $37^{\circ} \mathrm{C}$ for $16 \mathrm{~h}$. Following posthybridization washes in $1 \times$ SCC $/ 0.1 \%$ SDS at $50^{\circ} \mathrm{C}$, Cy5 and Cy3 hybridized signals were captured by ScanArray 5000 (GSI Lumonics, Packard BioScience, Pangbourne, UK). Raw images acquired were analyzed and quantified by the GenePix Pro 4.0 (Axon, Union City, CA, USA). Custom software Normalize Suite v1.56 was used for normalization of Cy3 and Cy5 intensities, data combination of dye swap experiments and integration of signal ratios determined with physical map locations of cDNAs in sequential order of megabase distances (http://www.utoronto.ca/cancyto/). ${ }^{27}$

\section{qRT-PCR}

Trizol extracted total RNA from cell lines, primary hepatocellular carcinoma and adjacent nonmalignant liver tissues was subjected to DNase treatment to eliminate possible carryover of genomic DNA. Control experiments by minus-RT-PCR were also performed to ensure RNA quality. First-strand cDNA was prepared from $2 \mu \mathrm{g}$ total RNA using random hexanucleotide primer and MultiScribe reverse transcriptase (Applied Biosystems, Foster City, CA, USA). Quantitative PCR (qPCR) was performed in triplicate assays using the TaqMan Universal Master Mix (Applied Biosystems). TaqMan assays targeting vitamin $\mathrm{D}$ binding protein precursor (GC, Hs00 167096_m1), alpha-2-plasmin inhibitor (SERPINF2, Hs00168686_m1), deleted in liver cancer 1 (DLC1, Hs00183436_m1), E-cadherin (CDH1, Hs00170423 _m1) fibrinogen-like 1 (FGL1, Hs00189514_m1), tumor protein p53 (TP53, Hs00153349_m1) and fibrinogen gamma peptide (FGG, Hs00241038_m1) were acquired (Applied Biosystems). TaqMan probes and primers for metallothionein 1G (MT1G) (GenBank accession number NM_005950) was designed using the Primer Express V2.0 software (forward primer 5'-TGCCGCTAGGTGTCT-3' ${ }^{\prime}$, reverse primer 5'-CGATGCCCCTTTGCAGAT-3', MGB probe $5^{\prime}$ FAM-CTGTGCCAAGTGTGC-3'). TaqMan gene expression assay for $18 \mathrm{~s}$ rRNA (Hs99999901_s1) was used as endogenous control. The two steps PCR conditions were $2 \mathrm{~min}$ at $50^{\circ} \mathrm{C}, 10 \mathrm{~min}$ at $95^{\circ} \mathrm{C}, 45$ cycles with $30 \mathrm{~s}$ at $95^{\circ} \mathrm{C}$, and $1 \mathrm{~min}$ at $55^{\circ} \mathrm{C}$. The amplification for the predesign assays was performed for 45 cycles with denaturation at $94^{\circ} \mathrm{C}$ for $30 \mathrm{~s}$, annealing at $60^{\circ} \mathrm{C}$ for $1 \mathrm{~min}$. The emission intensity was detected by the iCycler detection system (BioRad Laboratories, Hercules, CA, USA). Relative quantification values expressed as threshold cycle $\left(C_{\mathrm{t}}\right)$ were averaged and subsequently used to determine the relative expression ratios between cases. The median gene expressions of 8 normal livers were used for comparison. A no template negative control was also included in each experiment.

\section{Statistical Analysis}

The differences in gene expression levels between hepatocellular carcinoma tumors and surrounding nonmalignant livers were analyzed by the Wilcoxon signed rank test. The statistical significance between tumor stages was measured by the Mann-Whitney $U$-test. All analysis was performed with Prism software (GraphPad Software, San Diego, CA, USA). A $P$-value less than 0.05 was considered significant.

\section{Results}

\section{Downregulated Genes in Deletion Hotspots}

Gene expression profiling in primary tumors and cell lines indicated deregulated transcripts throughout the whole genome. Remarkably, profound repressions of distinct genes were indicated in common regions of allelic losses. Upregulated expressions, on the other hand, were less prominent and often associated with a high backgroundto-noise ratio. In 20 cell lines, 131 transcripts were found to be commonly downregulated, whereas frequent downregulations of 126 genes were suggested in 20 hepatocellular carcinoma tumors examined. To further define important genes, concordant transcripts that were repressed in both primary tumors and cell lines were scored. Using a median cutoff threshold of $>3$-fold reduction and an occurrence of $>50 \%, 35$ common candidate genes were indicated in both series (Table 1).

Alignment of cytogenetic information with expression profiling suggested the 35 genes located on 27 subchromosomal regions (Table 1). In particular, five genes resided within the deletion hotspots that have implicated in the early carcinogenetic events of hepatocellular carcinoma (Figure 1). These being vitamin D binding protein (GC) within smallest overlapping region 4q12-q23, fibrinogen gamma peptide (FGG) in 4q31-q35, FGL1 in 8p21-p22, 
Table 1 Common downregulated genes in hepatocellular carcinoma tumors and cell lines from transcriptional profiling

\begin{tabular}{|c|c|c|c|c|c|c|c|}
\hline \multirow[t]{2}{*}{ Cytoband } & \multirow{2}{*}{$\begin{array}{l}\text { Accession } \\
\text { no. }\end{array}$} & \multirow[t]{2}{*}{ Name } & \multirow[t]{2}{*}{ Symbol } & \multicolumn{2}{|c|}{20 Tumors } & \multicolumn{2}{|c|}{20 Cell lines } \\
\hline & & & & $\begin{array}{l}\text { Median fold } \\
\text { reduction }\end{array}$ & $\begin{array}{c}\text { Percentage } \\
\text { cases } \geq \\
3 \text {-fold }(\%)\end{array}$ & $\begin{array}{l}\text { Median fold } \\
\text { reduction }\end{array}$ & $\begin{array}{c}\text { Percentage } \\
\text { cases } \geq \\
3 \text {-fold }(\%)\end{array}$ \\
\hline $1 \mathrm{p} 13.3$ & W91952 & Vav 3 oncogene & $V A V 3$ & 3.50 & 53 & 3.48 & 67 \\
\hline 1p12 & BI918353 & $\begin{array}{l}\text { 3-hydroxy-3-methylglutaryl- } \\
\text { Coenzyme A synthase } 2\end{array}$ & HMGCS2 & 3.59 & 60 & 6.89 & 80 \\
\hline $1 \mathrm{q} 42.2$ & H22747 & Angiotensinogen & $A G T$ & 4.10 & 55 & 9.20 & 75 \\
\hline 2p24.1 & BG566740 & Apolipoprotein B & $A P O B$ & 3.59 & 60 & 3.24 & 50 \\
\hline $2 \mathrm{p} 22.2$ & W01373 & $\begin{array}{l}\text { CCAAT/enhancer binding } \\
\text { protein zeta }\end{array}$ & $C E B P Z$ & 4.28 & 60 & 3.76 & 75 \\
\hline $3 \mathrm{p} 24.2$ & W16685 & $N$-glycanase 1 & NGLY1 & 4.01 & 68 & 3.15 & 60 \\
\hline $3 q 21.3$ & H71112 & $\begin{array}{l}\text { MCM2 minichromosome } \\
\text { maintenance deficient } 2\end{array}$ & MCM2 & 4.70 & 65 & 5.58 & 74 \\
\hline $3 q 24-q 25.1$ & H86642 & Ceruloplasmin & $C P$ & 6.35 & 75 & 12.25 & 95 \\
\hline $3 q 25.1$ & T83911 & $\begin{array}{l}\text { Transmembrane } 4 \text { superfamily } \\
\text { member } 4\end{array}$ & TM4SF4 & 3.30 & 56 & 3.35 & 53 \\
\hline $3 q 25.32$ & W17370 & $\begin{array}{l}\text { G elongation factor, } \\
\text { mitochondrial } 1\end{array}$ & GFM1 & 5.08 & 77 & 28.71 & 100 \\
\hline *4q13.3 & R88884 & Vitamin D binding protein & GC & 7.37 & 74 & 10.15 & 67 \\
\hline${ }^{*} 4 \mathrm{q} 31.3$ & BG616563 & Fibrinogen gamma chain & $F G G$ & 11.79 & 93 & $\mathbf{5 2 . 5 4}$ & 100 \\
\hline 4q32.1 & H38897 & Tryptophan 2,3-dioxygenase & TDO2 & 3.06 & 50 & 4.56 & 67 \\
\hline $5 p 13.2$ & T96003 & LMBR1 domain containing 2 & LMBRD2 & 4.91 & 94 & 6.63 & 100 \\
\hline 6p21.33 & T87339 & Apolipoprotein M & APOM & 3.11 & 50 & 5.12 & 68 \\
\hline 6p12.2 & BE796134 & Glutathione $S$-transferase A2 & GSTA2 & 4.00 & 67 & 6.36 & 95 \\
\hline 7p15.3 & R69654 & Oxysterol binding protein-like 3 & OSBPL3 & 7.52 & 65 & 3.51 & 50 \\
\hline $7 q 22.1$ & R96774 & $\begin{array}{l}\text { Cytochrome } P 450 \text {, family } 3 \text {, } \\
\text { subfamily A, polypeptide } 7\end{array}$ & CYP3A7 & 5.85 & 56 & 13.87 & 92 \\
\hline${ }^{*} 8 \mathrm{p} 22-\mathrm{p} 21.3$ & BG618635 & Fibrinogen-like 1 & FGL1 & 4.31 & 53 & 10.88 & 80 \\
\hline $8 q 12.1$ & BM925604 & $\begin{array}{l}\text { RAB2, member RAS oncogene } \\
\text { family }\end{array}$ & $R A B 2$ & 3.33 & 62 & 3.16 & 55 \\
\hline $10 q 26.3$ & T97051 & $\begin{array}{l}\text { Mitochondrial short-chain } \\
\text { enoyl-coenzyme A }\end{array}$ & ECHS1 & 3.58 & 63 & 3.06 & 58 \\
\hline $11 \mathrm{p} 15.4$ & H12367 & Beta globin & $H B B$ & 5.44 & 74 & 7.64 & 93 \\
\hline $11 \mathrm{p} 15.1$ & H45773 & Serum amyloid A2 & $S A A 1$ & 3.10 & 55 & 3.77 & 65 \\
\hline $12 q 13.3$ & R35197 & Hydroxysteroid dehydrogenase & $\mathrm{RODH}$ & 5.02 & 58 & 12.31 & 95 \\
\hline $12 q 21.33$ & R87181 & Decorin & $D C N$ & 7.30 & 75 & 13.21 & 77 \\
\hline $12 q 24.13$ & BM805738 & Serine dehydratase & $S D S$ & 6.48 & 69 & 8.34 & 90 \\
\hline $14 q 12$ & N46702 & Syntaxin binding protein 6 & STXBP6 & 3.56 & 64 & 3.49 & 57 \\
\hline${ }^{*} 16 q 12.2$ & H57208 & Metallothionein 1G & MT1G & 9.29 & 75 & 3.10 & 60 \\
\hline${ }^{*} 17 p 13.3$ & H69261 & Alpha-2-plasmin inhibitor & SERPINF2 & 4.71 & $\mathbf{5 5}$ & 7.69 & 90 \\
\hline $17 q 11.2$ & H29155 & Vitronectin & $V T N$ & 11.96 & 75 & 7.23 & 78 \\
\hline $17 q 23.2$ & BM471342 & Myotubularin related protein 4 & MTMR4 & 7.17 & 93 & 5.45 & 80 \\
\hline $18 q 12.1$ & H25541 & Ring finger protein 138 & RNF138 & 12.15 & 68 & 7.55 & 95 \\
\hline $19 q 13.33$ & R08306 & Activating transcription factor 5 & ATF5 & 5.21 & 65 & 5.36 & 80 \\
\hline $20 \mathrm{p} 12.3$ & W91932 & Proliferating cell nuclear antigen & PCNA & 3.34 & 57 & 4.09 & 67 \\
\hline $20 q 11.23$ & H24115 & Transglutaminase 2 & TGM2 & 4.66 & 70 & 3.01 & 53 \\
\hline
\end{tabular}

*Bold represents candidate genes identified within the common deleted regions of hepatocellular carcinoma.

metallothionein 1G (MT1G) in 16q12.1-q23.1 and alpha-2-plasmin inhibitor (SERPINF2) in 17p13. A parallel comparative genomic hybridization analysis on 20 hepatocellular carcinoma cell lines indicated regional losses of 4q12-q23 and 4q31-q35 in 55\% (11/20 cell lines) and 75\% (15/20 cell lines) of cases, respectively. Regional chromosomal loss at 8p21p22 was found in $45 \%$ of cases (9/20 cell lines), whereas deletions on both 16q12-q23 and 17p13 were suggested in $30 \%$ (6/20 cell lines). A concordant downregulation of candidate genes and regional loss in cell lines was suggested in $65 \%$ for FGG, 45\% for GC and FGL1, 27\% for MT1G and $25 \%$ for SERPINF2.

\section{Validation in Cell Lines and Tumors}

The mRNA levels of GC, FGG, FGL1, MT1G and SERPINF2 were verified using the same array studied cell lines and 12 of the primary hepatocellular carcinomas. Although downregulations of DLC1, CDH1 and TP53 were not suggested from our array analysis, due to their reported tumorsuppressive role and their localization in the smallest overlapping regions, the mRNA expression levels of these genes were also investigated by qRT-PCR (Table 2). With the exception of DLC1 and CDH1, a repressed gene expression relative to pooled normal livers was confirmed in the 

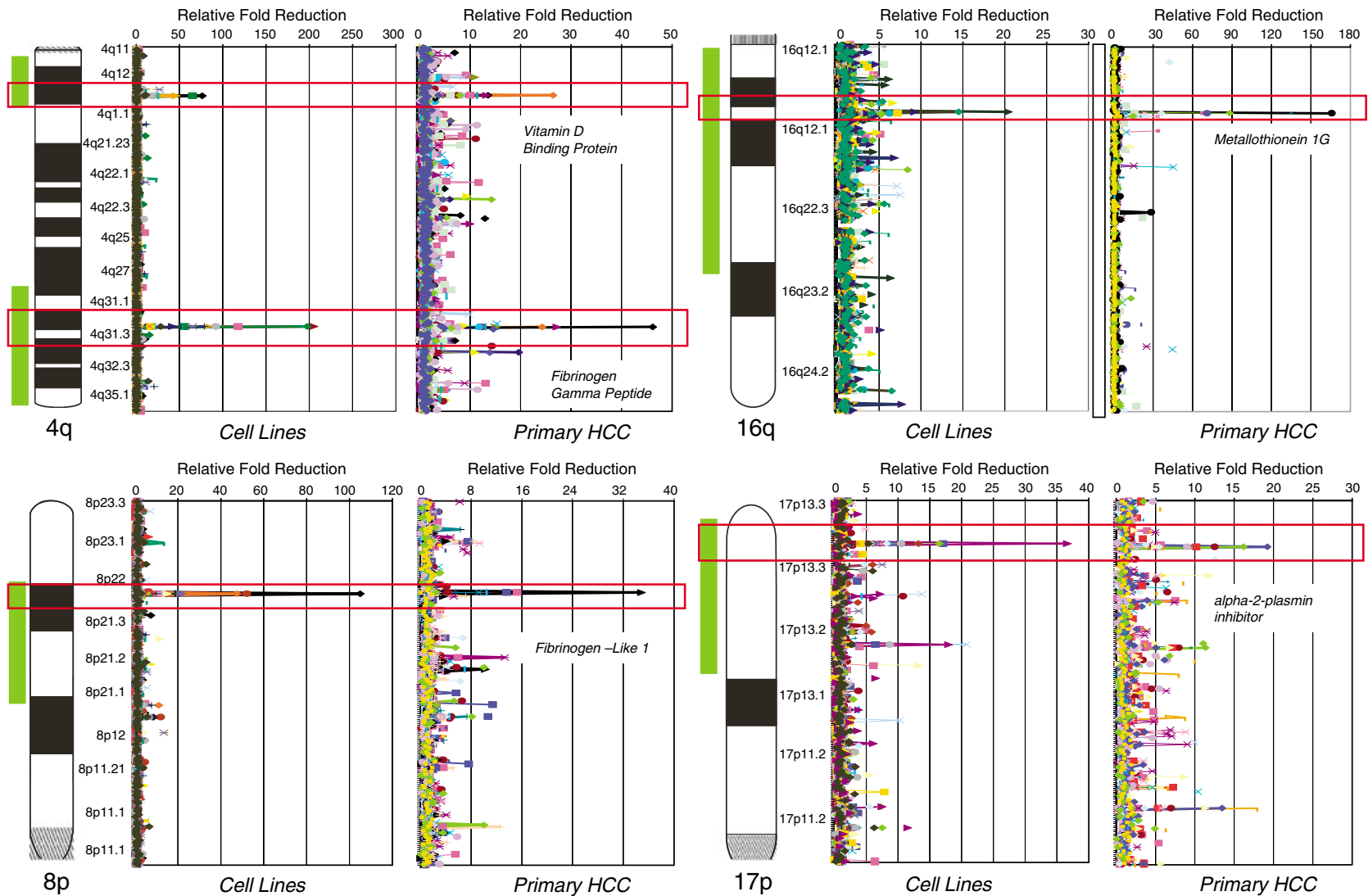

Figure 1 Positional expression profiling on chromosomes 4q, 8p, 16q and 17p in hepatocellular carcinoma cell lines and tumors. Relative expression levels as fold reductions determined for each transcript by microarray analysis on 20 hepatocellular carcinoma cell lines and 20 tumors were plotted along physical map location of each cDNA clone. Candidate genes that showed marked downregulations in more than $50 \%$ of both primary tumors and cell lines were highlighted by bracket. The smallest overlapping region of common losses was indicated as green bar next to the ideogram of each chromosome arm.

Table 2 Validation of candidate genes on hepatocellular carcinoma tumors and cell lines

\begin{tabular}{|c|c|c|c|c|c|}
\hline \multirow{2}{*}{$\begin{array}{l}\text { Chrom. } \\
\text { arm }\end{array}$} & \multirow[t]{2}{*}{ SOR } & \multirow[t]{2}{*}{ Genes, symbol } & \multirow[t]{2}{*}{ Location } & \multicolumn{2}{|c|}{ Expression ratio } \\
\hline & & & & Array (median, quartile) & qRT-PCR (median, quartile) \\
\hline \multirow[t]{2}{*}{$4 q$} & $4 q 12-q 13$ & Vitamin D binding protein, $G C$ & $4 q 13.3$ & $0.17(0.06-0.45)$ & $<0.01(<0.01-1.06)$ \\
\hline & $4 q 31-q 35$ & Fibrinogen gamma peptide, $F G G$ & $4 q 31.3$ & $0.04(0.01-0.09)$ & $0.50(<0.01-3.57)$ \\
\hline \multirow[t]{2}{*}{$8 p$} & $8 p 21-p 22$ & Fibrinogen-like 1, FGL1 & 8p22 & $0.10(0.05-0.40)$ & $0.68(<0.01-14.36)$ \\
\hline & & Deleted in liver cancer $1, D L C 1^{\mathrm{b}}$ & $8 p 22$ & $0.88(0.71-1.00)$ & $11.78(0.83-28.57)$ \\
\hline \multirow[t]{2}{*}{$16 q$} & $16 q 12.1-q 23.1$ & Metallothionein 1G, MT1G & $16 q 12.2$ & $0.27(0.14-0.42)$ & $<0.01(<0.01-0.02)$ \\
\hline & & E-cadherin, $C D H 1^{\mathrm{b}}$ & $16 q 22.1$ & $1.03(0.89-1.61)$ & $1.64(0.17-12.56)$ \\
\hline \multirow[t]{2}{*}{$17 p$} & $17 \mathrm{p} 13$ & $\begin{array}{l}\text { Alpha-2-plasmin inhibitor, } \\
\text { SERPINF2 }\end{array}$ & $17 \mathrm{p} 13.3$ & $0.16(0.10-0.33)$ & $0.10(<0.01-0.65)$ \\
\hline & & Tumor protein p53, TP53 & $17 \mathrm{p} 13.1$ & $1.22(1.04-1.53)$ & $0.01(<0.01-0.47)$ \\
\hline
\end{tabular}

${ }^{a}$ qRT-PCR validations included 20 cell lines and 12 primary tumors that were subjected to microarray analysis.

${ }^{\mathrm{b}}$ Known TSG located within region of frequent allelic losses found in HCC.

majority of specimens examined (Table 2). A downregulation of FGG was suggested in $60 \%$ of cases at 0.50 -fold $(<0.01-3.57)$ (median, quartiles) and FGL1 in 53\% at 0.68 -fold $(<0.01-14.36)$. Repressed GC and MT1G expressions at less than 0.01-fold were suggested in $70 \%$ of cases and $97 \%$, respectively. On 17p13, downregulations of SERPINF2 were suggested in $84 \%$ of cases at 0.10 -fold $(<0.01-0.65)$ and TP53 in $88 \%$ at $0.01 \quad(<0.01-$ 0.47) (Table 2). 
Table 3 Expression of array-derived candidate genes in primary hepatocellular carcinoma

\begin{tabular}{llcc}
\hline SOR & Genes, symbol & Ratio of tumor to paired adjacent liver \\
\cline { 3 - 4 } & & Percentage cases (\%) & Median (quartiles) \\
\hline 4q12-q13 & Vitamin D binding Protein, GC & 20 & $0.12(0.25-0.86)$ \\
4q31-q35 & Fibrinogen gamma peptide, FGG & 30 & $0.19(0.02-0.32)$ \\
8p21-p22 & Fibrinogen like 1, FGL1 & 23 & $0.009(0.08-0.63)$ \\
16q12.1-q23.1 & Metallothionein 1G, MT1G & 63 & $0.01(0.00-0.16)$ \\
17p13 & Alpha-2-plasmin inhibitor, SERPINF2 & 33 & 0.0156 \\
& & & 0.0001 \\
\end{tabular}

\section{Investigation in Primary Hepatocellular Carcinoma}

The mRNA expression levels of FGG, GC, FGL1, MT1G and SERPINF2 were further examined in primary HCC tumors and adjacent nontumorous livers by qRT-PCR. In 30 cases examined, downregulation of MT1G expression was most prominent with more than100-fold reductions indicated in $63 \%$ of tumors $(P=0.0001)$ (Table 3$)$. A significant reduction of FGG, FGL1 and SERPINF2 in tumors was also suggested (Table 3). Statistical analysis of GC, FGG, FGL1, MT1G and SERPINF2 expressions between early (T1/2) and advanced (T3/T4) tumor stages did not suggest differences with disease progression $(P>0.05)$ In our series of hepatocellular carcinoma studied, five cases arose from a noncirrhotic background. The adjacent nontumorous liver of these five noncirrhotic tumors demonstrated expressions of FGG and FGL1 similar to that found in normal livers. For GC, MT1G and SERPINF2, on the other hand, a downregulation of these genes was suggested. Nevertheless, the median expressions of all tested genes were comparable between the cirrhotic and noncirrhotic hepatocellular carcinoma tumors.

\section{Discussion}

In the present microarray study, early passages and established hepatocellular carcinoma cell lines have been utilized as a homogenous source of malignant hepatocytes to elucidate for differentially expressed transcripts in hepatocellular carcinoma. Results derived were further evaluated against genes determined from primary tumors, which indicated 35 candidates to be commonly downregulated in both cell lines and tumors. As diminutions on 4q12-q23, 4q31-q35, 8p21-p22, 16q12.1-q23.1 and 17p13 have been suggested in the early neoplastic changes of hepatocellular carcinoma, ${ }^{9,28-30}$ it was interesting to note that five consistently downregulated genes, FGG, GC, FGL1, MT1G and SERPINF2, were consistently downregulated in these characteristic regions of allelic losses. Parallel analysis by comparative genomic hybridization on the 20 hepatocellular carcinoma cell lines studied indicated a high concordance of regional loss and downregulations of FGG, GC and FGL1, while downregulations of MT1G and SERPINF2 were more frequent than cell lines harboring chromosomal losses. This discrepancy may be explained by the presence of other inactivation mechanisms, such as hypermethylation at the promoter region, that play a role in the control of gene expressions. Moreover, our group has recently shown that the downregulation of a novel gene ACP5 on chromosome 19p13 was associated with chromosomal breakage, which might represent an alternate inactivation mechanism. ${ }^{25}$ From our array analysis, a reduced expression of the proliferating cell nuclear antigen (PCNA) gene was suggested in both hepatocellular carcinoma cell lines and tumors, which would seem contrary to a recent study conducted by Chen et $a l^{31}$ One possible explanation for this discrepancy would be the cDNA clone (IMAGE clone: 415202) representing the PCNA gene on the Ontario array has an almost $90 \%$ sequence homology to a region located on chromosome X. The hybridization signal may therefore be one of the false positive errors that are commonly found in microarray studies. Nevertheless, in line with our findings, downregulations of FGG, FGL1, SERPINF2 and MT1G were also observed in the hepatocellular carcinoma tumor relative to the nontumorous liver in the Chen et al study. ${ }^{32}$

According to qPCR, the expression levels of FGG, GC, FGL1, MT1G and SERPINF2 were similar in early and advanced stages hepatocellular carcinoma. This might in turn suggest that the inactivation of these genes occurred early in the tumor development and have been maintained through progression. Moreover, FGL1 was more profoundly repressed than another tumor-suppressor gene DLC1 located on the same $8 p 22$ region. This was also found for MT1G and SERPINF2, which displayed more repressed expressions than wellknown tumor-suppressor genes CDH1 and TP53 within the same physical location. Our finding may hence potentially underline a value for these novel candidates in hepatocellular carcinoma development.

Allelic losses on $4 \mathrm{q}$ are common in hepatocellular carcinoma, although targeted genes in the smallest overlapping regions have not been suggested. In this study, we report on the novel finding of repressed FGG (on 4q31.3) expressions in hepatocellular 
carcinoma tumors, despite this protein is primarily synthesized in the liver. ${ }^{33}$ A downregulation of FGG was found in $95 \%$ of cases compared to normal livers (Table 2), although in comparison to their adjacent nontumorous cirrhotic livers significant reduction was suggested in only $30 \%$ (Table 3 ). This observation was also noted for other candidate genes examined (Tables 2 and 3). Our findings may therefore be interpreted as aberrant gene expressions are also present in the adjacent putative premalignant cirrhotic liver, and may have constituted to the early tumor development. Deposition of fibrin(ogen) into the extracellular matrix serves as a scaffold to support adhesion, binding to growth factors and facilitates migration during angiogenesis. ${ }^{34}$ Early cirrhotic hepatocellular carcinoma nodules are always surrounded by a sheath of fibrotic tissue deposited during the course of chronic infection. The early loss of the FGG in a cirrhotic liver could have modulated the outgrowth of early hepatocellular carcinoma by reducing the integrity of extracellular matrix, and promoted cell growth by increasing the elasticity of matrix structure. Besides an important element in blood coagulation, FGG is also a structural component of fibrinogen E-fragment. Studies on endothethial cells have demonstrated fibrinogen E-fragment as a potent inhibitor of angiogenesis that can inhibit cell migration and tubule formation induced from strong proangiogenic factors such as VEGF and bFGF. ${ }^{35}$ This inhibition is believed to have occurred through the binding of fibrinogen E-fragment at postreceptor locus that is common to both VEGF and bFGF. ${ }^{35}$ It is therefore plausible that the loss of the FGG chain could lead to structural alterations of fibrinogen E-fragment resulting in lost or reduced binding inhibition on the growth factors related angiogenesis activity and consequently early tumor growth.

In the context of extracellular matrix, it was also particularly interesting to have identified common downregulations of SERPINF2 (also known as alpha-2-anti-plasmin). SERPINF2 is the major inhibitor of the proteolytic enzyme plasmin that digests fibrins. ${ }^{36,37}$ The plasmin activation system is known to play a key role in extracellular matrix degradation. ${ }^{38}$ The reduced expressions of SERPINF2 might lead to an increased level of activated plasmin, which could impair the stabilization of fibrin bundle and in turn the integrity of liver extracellular matrix. In addition, FGL1 (also named as LFIRE-1/HFREP-1) is also a member of the fibrinogen superfamily ${ }^{39}$ and a liver-specific gene that is frequently downregulated in hepatocellular carcinoma at both the mRNA and protein levels. ${ }^{40}$ Upon restoration of exogenous wild-type FGL1 expression in hepatocellular carcinoma, functional examinations have indicated an inhibitory effect of FGL1 on cell proliferation, anchorage-dependent or independent growth in vitro, and suppression of tumorigenicity in athymic nude mice. ${ }^{40}$ Besides displaying tumor-suppressive characteristics, since downregulations of FGL1 expression in hepato cellular carcinoma is frequently association with $8 \mathrm{p}$ allelic losses, it has been further implicated as a tumor-suppressor gene on $8 \mathrm{p}^{40}$

Previous microarray studies have showed that several isoforms of metallothionein such as MT1A and MT1F are frequently downregulated in hepatocellular carcinoma compared to nontumorous livers. ${ }^{41-43}$ However, none of these studies has attempted validation analysis. In this study, our array finding concurred with qRT-PCR verifications, which also indicated the repressions of MT1G corresponded to the highest incidence $(63 \%)$ and magnitude in hepatocellular carcinoma tumors relative to their adjacent nonmalignant liver tissue $(P=0.0001)$ (Table 3).

In conclusion, utilizing microarray technology as a tool, we have successfully identified a number of candidate genes throughout the hepatocellular carcinoma genome and within the deletion hotspots. As number of genes found including SERPINF2, FGG, Decorin, Vitronectin (Table 1) are structural components of the extracellular matrix, we postulate that common downregulations of these targets may perturb the integrity or maintenance of extracellular matrix. Indeed, breakdown of the extracellular matrix is contributory to cancer developments. ${ }^{44}$ Moreover, extracellular matrix also serves as a reservoir of growth factors that can modulate cell morphology and proliferation. ${ }^{44,45}$ Thus, deregulation of extracellular matrix components may have adverse effects on homeostasis, which consequently enhances local tumor cells growth and promotes hepatocellular carcinoma formation.

\section{References}

1 Okuda K. Hepatocellular carcinoma. J Hepatol 2000; 32(Suppl 1):225-237.

2 Lau WY. Primary liver tumors. Semin Surg Oncol 2000; 19:135-144

3 Lai EC, Fan ST, Lo CM, et al. Hepatic resection for hepatocellular carcinoma. An audit of 343 patients. Ann Surg 1995;221:291-298.

4 Wong N, Lai P, Lee SW, et al. Assessment of genetic changes in hepatocellular carcinoma by comparative genomic hybridization analysis: relationship to disease stage, tumor size, and cirrhosis. Am J Pathol 1999; 154:37-43.

5 Marchio A, Meddeb M, Pineau P, et al. Recurrent chromosomal abnormalities in hepatocellular carcinoma detected by comparative genomic hybridization. Genes Chromosomes Cancer 1997;18:59-65.

6 Kusano N, Shiraishi K, Kubo K, et al. Genetic aberrations detected by comparative genomic hybridization in hepatocellular carcinomas: their relationship to clinicopathological features. Hepatology 1999;29: 1858-1862.

7 Yuan BZ, Miller MJ, Keck CL, et al. Cloning, characterization, and chromosomal localization of a gene frequently deleted in human liver cancer (DLC-1) homologous to rat RhoGAP. Cancer Res 1998;58: 2196-2199. 
8 Lau SH, Guan XY. Cytogenetic and molecular genetic alterations in hepatocellular carcinoma. Acta Pharmacol Sin 2005;26:659-665.

9 Zondervan PE, Wink J, Alers JC, et al. Molecular cytogenetic evaluation of virus-associated and nonviral hepatocellular carcinoma: analysis of 26 carcinomas and 12 concurrent dysplasias. J Pathol 2000; 192:207-215.

10 Yeh SH, Chen PJ, Lai MY, et al. Allelic loss on chromosomes $4 \mathrm{q}$ and $16 \mathrm{q}$ in hepatocellular carcinoma: association with elevated alpha-fetoprotein production. Gastroenterology 1996;110:184-192.

11 Chang J, Kim NG, Piao Z, et al. Assessment of chromosomal losses and gains in hepatocellular carcinoma. Cancer Lett 2002;182:193-202.

12 Yasui K, Arii S, Zhao C, et al. TFDP1, CUL4A, and CDC16 identified as targets for amplification at 13q34 in hepatocellular carcinomas. Hepatology 2002;35: 1476-1484.

13 Chow LS, Lo KW, Kwong J, et al. RASSF1A is a target tumor suppressor from $3 \mathrm{p} 21.3$ in nasopharyngeal carcinoma. Int J Cancer 2004;109:839-847.

14 Wong TS, Tang KC, Kwong DL, et al. Differential gene methylation in undifferentiated nasopharyngeal carcinoma. Int J Oncol 2003;22:869-874.

15 Kok K, Naylor SL, Buys CH. Deletions of the short arm of chromosome 3 in solid tumors and the search for suppressor genes. Adv Cancer Res 1997;71:27-92.

16 Qiu GH, Tan LK, Loh KS, et al. The candidate tumor suppressor gene BLU, located at the commonly deleted region 3p21.3, is an E2F-regulated, stress-responsive gene and inactivated by both epigenetic and genetic mechanisms in nasopharyngeal carcinoma. Oncogene 2004;23:4793-4806.

17 Alon U, Barkai N, Notterman DA, et al. Broad patterns of gene expression revealed by clustering analysis of tumor and normal colon tissues probed by oligonucleotide arrays. Proc Natl Acad Sci USA 1999;96: 6745-6750.

18 Nagai H, Terada Y, Tajiri T, et al. Characterization of liver-cirrhosis nodules by analysis of gene-expression profiles and patterns of allelic loss. J Hum Genet 2004; 49:246-255.

19 Okabe H, Satoh S, Kato T, et al. Genome-wide analysis of gene expression in human hepatocellular carcinomas using cDNA microarray: identification of genes involved in viral carcinogenesis and tumor progression. Cancer Res 2001;61:2129-2137.

$20 \mathrm{Xu} \mathrm{XR}$, Huang J, Xu ZG, et al. Insight into hepatocellular carcinogenesis at transcriptome level by comparing gene expression profiles of hepatocellular carcinoma with those of corresponding noncancerous liver. Proc Natl Acad Sci USA 2001;98:15089-15094.

21 Lee JS, Thorgeirsson SS. Genetic profiling of human hepatocellular carcinoma. Semin Liver Dis 2005;25: 125-132.

22 Wong N, Chan KY, Macgregor PF, et al. Transcriptional profiling identifies gene expression changes associated with IFN-alpha tolerance in hepatitis C-related hepatocellular carcinoma cells. Clin Cancer Res 2005;11: 1319-1326.

23 Pang E, Wong N, Lai PB, et al. Consistent chromosome 10 rearrangements in four newly established human hepatocellular carcinoma cell lines. Genes Chromosomes Cancer 2002;33:150-159.

24 Pang E, Wong N, Lai PB, et al. A comprehensive karyotypic analysis on a newly developed hepatocel- lular carcinoma cell line, HKCI-1, by spectral karyotyping and comparative genomic hybridization. Cancer Genet Cytogenet 2000;121:9-16.

25 Chan KY, Wong N, Lai PB, et al. Transcriptional profiling on chromosome 19p indicated frequent downregulation of ACP5 expression in hepatocellular carcinoma. Int J Cancer 2005;114:902-908.

26 Fleming ID. American Cancer Society, American College of Surgeons, National Cancer Institute (US), American Joint Committee on Cancer, American College of Radiology, College of American Pathologists. AJCC Cancer Staging Manual, 5th edn. Lippincott-Raven: Philadelphia, 1997, XV, 294pp.

27 Beheshti B, Braude I, Marrano P, et al. Chromosomal localization of DNA amplifications in neuroblastoma tumors using cDNA microarray comparative genomic hybridization. Neoplasia 2003;5:53-62.

28 Lin YW, Sheu JC, Huang GT, et al. Chromosomal abnormality in hepatocellular carcinoma by comparative genomic hybridisation in Taiwan. Eur J Cancer 1999;35:652-658.

29 Okabe H, Ikai I, Matsuo K, et al. Comprehensive allelotype study of hepatocellular carcinoma: potential differences in pathways to hepatocellular carcinoma between hepatitis $B$ virus-positive and -negative tumors. Hepatology 2000;31:1073-1079.

30 Zhang SH, Cong WM, Xian ZH, et al. Clinicopathological significance of loss of heterozygosity and microsatellite instability in hepatocellular carcinoma in China. World J Gastroenterol 2005;11:3034-3039.

31 Chen ZM, Crone KG, Watson MA, et al. Identification of a unique gene expression signature that differentiates hepatocellular adenoma from well-differentiated hepatocellular carcinoma. Am J Surg Pathol 2005; 29:1600-1608.

32 Rhodes DR, Yu J, Shanker K, et al. ONCOMINE: a cancer microarray database and integrated data-mining platform. Neoplasia 2004;6:1-6.

33 Straub PW. A study of fibrinogen production by human liver slices in vitro by an immunoprecipitin method. J Clin Invest 1963;42:130-136.

34 Simpson-Haidaris PJ, Rybarczyk B. Tumors and fibrinogen. The role of fibrinogen as an extracellular matrix protein. Ann NY Acad Sci 2001;936:406-425.

35 Bootle-Wilbraham CA, Tazzyman S, Marshall JM, et al. Fibrinogen E-fragment inhibits the migration and tubule formation of human dermal microvascular endothelial cells in vitro. Cancer Res 2000;60: 4719-4724.

36 Lee KN, Jackson KW, Christiansen VJ, et al. A novel plasma proteinase potentiates alpha2-antiplasmin inhibition of fibrin digestion. Blood 2004;103: 3783-3788.

37 Owensby DA, Morton PA, Wun TC, et al. Binding of plasminogen activator inhibitor type-1 to extracellular matrix of Hep G2 cells. Evidence that the binding protein is vitronectin. J Biol Chem 1991; 266:4334-4340.

38 Dano K, Behrendt N, Hoyer-Hansen G, et al. Plasminogen activation and cancer. Thromb Haemost 2005;93: 676-681.

39 Yamamoto T, Gotoh M, Sasaki H, et al. Molecular cloning and initial characterization of a novel fibrinogen-related gene, HFREP-1. Biochem Biophys Res Commun 1993;193:681-687.

40 Yan J, Yu Y, Wang N, et al. LFIRE-1/HFREP-1, a liverspecific gene, is frequently downregulated and has 
growth suppressor activity in hepatocellular carcinoma. Oncogene 2004;23:1939-1949.

41 lizuka N, Oka M, Yamada-Okabe $\mathrm{H}$, et al. Selforganizing-map-based molecular signature representing the development of hepatocellular carcinoma. FEBS Lett 2005;579:1089-1100.

42 Iizuka N, Oka M, Yamada-Okabe H, et al. Comparison of gene expression profiles between hepatitis $\mathrm{B}$ virusand hepatitis $\mathrm{C}$ virus-infected hepatocellular carcinoma by oligonucleotide microarray data on the basis of a supervised learning method. Cancer Res 2002;62: 3939-3944.
$43 \mathrm{Lu}$ DD, Chen YC, Zhang XR, et al. The relationship between metallothionein-1F (MT1F) gene and hepatocellular carcinoma. Yale J Biol Med 2003;76:55-62.

44 Shekhar MP, Pauley R, Heppner G. Host microenvironment in breast cancer development: extracellular matrix-stromal cell contribution to neoplastic phenotype of epithelial cells in the breast. Breast Cancer Res 2003;5:130-135.

45 Getzenberg RH, Pienta KJ, Huang EY, et al. Modifications of the intermediate filament and nuclear matrix networks by the extracellular matrix. Biochem Biophys Res Commun 1991;179:340-344. 